\title{
含尿嘧啶结构单元二肽衍生物的设计、合成及生物活性研究
}

\author{
唐雪梅 $a, b$ 范莉 ${ }^{a}$ 张泽朝 ${ }^{a}$ 杨大成*, $a$ \\ $\left({ }^{a}\right.$ 西南大学化学化工学院 重庆 400715) \\ ( $b$ 西南大学生命科学学院 重庆 400715)
}

\begin{abstract}
摘要 为探索新型抗糖尿病分子, 设计了含有尿嘧啶结构单元的二肽衍生物. 以尿嘧啶、多聚甲醛和半胱氨酸为原料, 经过两步反应获得关键中间体 $S$-胸腺嘧啶- $L$-半胱氨酸(IM-2), 再经氨基保护、羧基酯化和氨基酸偶联, 顺利合成了 16 个二肽衍生物. 所得目标化合物均经 ${ }^{1} \mathrm{H} N \mathrm{NM} 、{ }^{13} \mathrm{C} \mathrm{NMR}$ 和 HRMS 进行结构确认, 并开展了过氧化物酶体增殖物受体 反应元件(PPRE)激动活性、 $\alpha$-葡萄糖苷酶-rat 抑制活性、二肽基肽酶-4 (DPP-4)抑制活性篮选. 生物活性结果显示, 这些 二肽衍生物的 PPRE 相对激动活性、 $\alpha$-葡萄糖苷酶和 DPP-4 抑制活性都很弱; 同时发现，该类分子的 $\alpha$-葡萄糖苷酶抑 制活性变化趋势与 PPRE 激动活性、DPP-4 抑制活性变化趋势相反, 这为新型多肽多靶点药物的设计提供了新思路.

关键词 糖尿病; 尿嘧啶; 二肽衍生物; 过氧化物酶体增殖物激活受体反应元件(PPRE); $\alpha$-葡萄糖苷酶抑制活性; 二肽 基肽酶-4 抑制活性
\end{abstract}

\section{Design, Synthesis and Biological Activity of Dipeptide Derivatives Bearing Uracil Unit}

\author{
Tang, Xuemei ${ }^{a, b} \quad$ Fan, $\mathrm{Li}^{a} \quad$ Zhang, Zechao ${ }^{a} \quad$ Yang, Dacheng ${ }^{*}, a$ \\ ( ${ }^{a}$ School of Chemistry and Chemical Engineering, Southwest University, Chongqing 400715) \\ ( ${ }^{b}$ School of Life Sciences, Southwest University, Chongqing 400715)
}

\begin{abstract}
In order to explore a new type of anti-diabetic molecules, dipeptide derivatives containing uracil structural units were designed. The key intermediate $S$-thymine- $L$-cysteine (IM-2) was obtained from uracil, paraformaldehyde and cysteine through two step reactions, and then 16 dipeptide derivatives were successfully synthesized through amino protection, carboxylation and amino acid coupling. All new compounds have been characterized by ${ }^{1} \mathrm{H} N M R,{ }^{13} \mathrm{C}$ NMR and HRMS, and the peroxisome proliferator response element (PPRE) activated activity, $\alpha$-glucosidase-rat inhibitory activity and dipeptidyl peptidase-4 (DPP-4) inhibitory activity were screened for all target molecules. The results showed that these molecules had weak above-mentioned activities, meanwhile the change trend of $\alpha$-glucosidase-rat inhibitory activity of these molecules is opposite to that of PPRE agonistic activity and DPP-4 inhibitory activity. It maybe provides an idea for the research of designing novel polypeptide multi-target drugs.

Keywords diabetes; uracil; dipeptide derivative; peroxisome proliferator response element (PPRE); $\alpha$-glucosidase-rat inhibitory activity; dipeptidyl peptidase-4 inhibitory activity
\end{abstract}

糖尿病是一种以高血糖症为特征的慢性代谢性疾 病，可能导致心血管疾病、肾衰竭、神经病变、失明和 中风等多种并发症 ${ }^{[1,2]}$. 根据国际糖尿病联合会 2017 年 的统计, 大约有 4.25 亿人患有因胰岛素分泌缺乏或胰岛 素抵抗引起的 II 型糖尿病 ${ }^{[3]}$, 且患病人数还在不断增加, 糖尿病已成为全球公共卫生威胁. 目前 II 型糖尿病的治
疗药物一般包括磺嫝类、二甲双胍类、噻唑烷二酮类 (thiazolidinediones, TZD)、 $\alpha$-葡萄糖苷酶抑制剂、DPP-4 抑制剂、胰高血糖素样肽-1 (GLP-1)抑制剂和钠-葡萄糖 协同转运蛋白 2 (SGLT-2)抑制剂等 ${ }^{[4,5]}$. 虽然这些临床药 物能有效地降低患者的空腹血糖浓度, 但由于其副作 用，如肥胖、心血管疾病、心律失常、胃肠不耐受、心

\footnotetext{
* Corresponding author. E-mail: hxydc@swu.edu.cn

Received November 30, 2018; revised January 26, 2019; published online February 19, 2019.

Project supported by the National Natural Science Foundation of China (No. 21542003) and the Natural Science Foundation of Chongqing City (No. cstc2016jcyjA0421).

国家自然科学基金(No. 21542003)和重庆市自然科学基金(No. cstc2016jcyjA0421)资助项目.
} 
肌梗死及给药方式(如注射)等限制了其临床使用 ${ }^{[6 \sim 13]}$, 因此仍然迫切需要开发新型抗糖尿病药物 ${ }^{[14]}$.

多肽药物多数由人体必需的氨基酸组成, 具有毒性 小、安全性高等独特优点. 抗菌肽、抗肿瘤多肽、多肽 疫苗等多肽药物目前已被广泛应用. 在慢性病治疗领 域, 比如自身免疫性疾病、高血压、糖尿病、骨质疏松 等, 多肽药物的优势日益明显 ${ }^{[14]}$, 已成为创新药物研发 的热点之一 ${ }^{[15]}$. GLP-1 受体激动剂艾塞那肽(exenatide)、 利司那肽(lixisenatide)和利拉鲁肽(liraglutide)都是多肽 分子, 已被美国食品药品监督管理局(FDA)批准用于 II 型糖尿病的治疗. 研究发现, 多肽类分子 $\mathbf{A}$ 具有较好的 蛋白酪氨酸磷酸酶 $1 \mathrm{~B}$ (PTP-1B) 抑制作用 $\left(\mathrm{IC}_{50}\right.$ 为 2.9 $\left.\mu \mathrm{mol} \cdot \mathrm{L}^{-1}\right)$, 显示出一定的抗糖尿病活性 ${ }^{[16]}$; 以肉桂醛 为母体的三肽衍生物 $\mathbf{B}^{[17]}$ 和二肽衍生物 $\mathbf{C}^{[18]}$ 具有显著的 抗糖尿病活性(图 1). 由此可见, 研发新型多肽类抗糖尿 病药物具有重要的发展前景.<smiles>CC(C)CC(CC(=O)[C@H](Cc1ccc(OCC(=O)O)cc1)NC(=O)[C@H](CCC(=O)OCc1ccccc1)NC(F)(F)F)C(N)=O</smiles><smiles>NC(=O)C(CCC(=O)O)NC(=O)[C@H](CCC(=O)O)NC(=O)CNC(=O)c1ccc(/C=C/C=O)cc1</smiles><smiles>[R]N[C@H](Cc1ccccc1)C(=O)N[C@@H](Cc1ccc(OCC(=O)O)c(-c2nn[nH]n2)c1)C(=O)NCCCCC</smiles>

图 1 具有抗糖尿病活性的多肽分子举例

Figure 1 Examples of polypeptide molecules with anti-diabetic activity

尿嘧啶是生物体的五种必需天然碱基之一, 在生物 体中有着重要的作用. 5-氟尿嘧啶是一种有效的抗代谢 抗肿瘤药; 尿嘧啶是普洱茶降糖降脂活性成分中的重要 辅助成分之一 ${ }^{[19]}$; 含有尿嘧啶结构单元的 DPP-4 抑制剂 SYR-322 (Alogliptin) 是获得 FDA 批准的第 4 个 DPP-4
抑制剂，是高选择性和强靶向特异性的口服降糖药 物 ${ }^{[20]}$; 含有尿嘧啶结构单元的化合物 D 也具有较强的 DPP-4 抑制活性(图 2) ${ }^{[21]}$. 这些表明，尿嘧啶结构单元可 能是抗糖尿病活性分子的重要结构单元. 受此启发, 我 们尝试将尿嘧啶结构单元引入新的目标分子之中, 希望 获取新型的抗糖尿病活性分子.<smiles>Cn1c(=O)cc(N2CCC[C@H](N)C2)n(Cc2ccccc2C#N)c1=O</smiles>

SYR-322 (Alogliptin)<smiles>[R]C(=O)[Y9]1cc(Cn2c(=O)cc(N3CCC[C@@H](N)C3)n(C#CC)c2=O)nn1</smiles>

D
图 2 含有尿嘧啶结构单元的抗糖尿病分子

Figure 2 Anti-diabetes molecules containing uracil unit

在将尿嘧啶引入肽核酸时, 余孝其等 ${ }^{[22]}$ 采用半胱 氨酸侧链的放基与羟甲基化的尿嘧啶连接形成肽核酸 单体的策略，合成了许多肽核酸分子. 作者 ${ }^{[23 ~ 25]}$ 借鉴该 合成思路并通过改进，高收率地合成了含有尿嘧啶结构 单元的肽核酸单体; 将该单体引入多肽分子中, 设计并 合成了含有尿嘧啶碱基的多肽衍生物. 作为研究的探索 阶段，作者设计了尿嘧啶碱基修饰的二肽衍生物 $\mathbf{T M}$ (Scheme 1), 希望从中获得具有抗糖尿病活性的先导分 子, 为后续研究提供新的思路.

\section{1 结果与讨论}

\section{1 化合物的合成}

采用改进的合成方法, 以尿嘧啶和多聚甲醛为原 料，在强碱中分段(不同温度)反应，能以很高收率得到 5-羟甲基尿嘧啶 (5-HmU); 5-HmU 与半胱氨酸侧链巯基 连接, 得到关键中间体 $S$-胸腺嘧啶 $-L$ - 半胱氨酸 (IM-2) $)^{[23 \sim 25]}$; IM-2 经氨基保护获得中间体 IM-3 ${ }^{[26,27]}$; 采用二氯亚砜和甲醇法 ${ }^{[23,28 ~ 31]}$, 一步反应将 IM-3 的羧 基甲酯化和氨基脱 Boc, 得到肽核酸单体 IM-4 ${ }^{[23]}$; IM-4 与 Fmoc 保护的氨基酸偶联 ${ }^{[26,27,32]}$, 即得到目标分子 TM. 本研究合成了 16 个目标分子(表 1), 结构均经过 ${ }^{1} \mathrm{H}$ NMR, ${ }^{13} \mathrm{C}$ NMR 和 HRMS 确认.

实验发现，中间体 IM-2 在反应液中有一定溶解度. 为了得到尽可能多的产品, 需要对滤液多次浓缩; 浓缩 之前一定要先中和一部分酸, 以避免浓缩后酸性太强而 导致氨基酸的消旋和其它不必要的副反应，同时尽量避 免产品包裹无机盐.

目标分子 $\mathbf{T M}$ 的合成采用液相多肽合成中的 DCC/ HOBt 或 DIC/HOBt 偶联法 ${ }^{[26,27,32]}$. 实验表明, 反应时间 和碱对反应有较大影响. 首先, 反应体系中需加入有机 


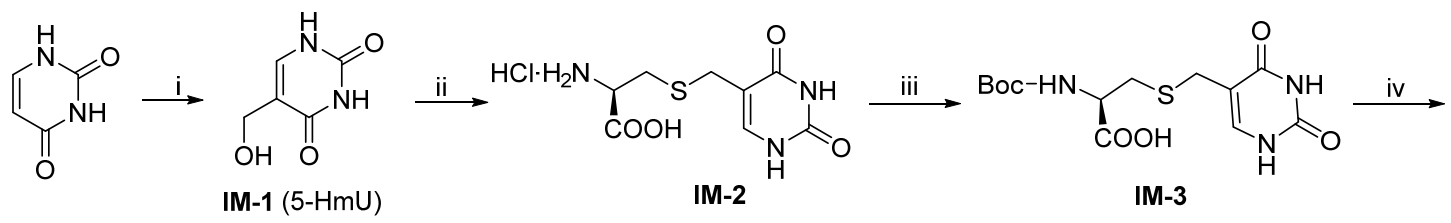<smiles>[R]C(NC(=O)OCC1c2ccccc2-c2ccccc21)C(=O)NC(CSCc1c[nH]c(=O)[nH]c1=O)C(=O)OC</smiles>

$\mathrm{R}=\mathrm{CH}_{3}(\mathbf{a}), \mathrm{CH}_{2} \mathrm{C}_{6} \mathrm{H}_{5}(\mathbf{b}), \mathrm{CH}\left(\mathrm{CH}_{3}\right)_{2}(\mathbf{c}), \mathrm{CH}_{2} \mathrm{CH}\left(\mathrm{CH}_{3}\right)_{2}(\mathbf{d}), \mathrm{CH}\left(\mathrm{CH}_{3}\right) \mathrm{CH}_{2} \mathrm{CH}_{3}(\mathbf{e}), \mathrm{CH}\left(\mathrm{CH}_{3}\right) \mathrm{OC}\left(\mathrm{CH}_{3}\right)_{3}(\mathbf{f}), \mathrm{CH}_{2} \mathrm{COOC}\left(\mathrm{CH}_{3}\right)_{3}(\mathbf{g})$, $\left(\mathrm{CH}_{2}\right)_{4} \mathrm{NHCOOC}\left(\mathrm{CH}_{3}\right)_{3}(\mathbf{h}),\left(\mathrm{CH}_{2}\right)_{2} \mathrm{SCH}_{3}(\mathbf{i}),\left(\mathrm{CH}_{2}\right)_{3}(\mathrm{j}), \mathrm{CH}_{2} \mathrm{C}_{6} \mathrm{H}_{4} \mathrm{OC}\left(\mathrm{CH}_{3}\right)_{3}(\mathbf{k}),\left(\mathrm{CH}_{2}\right)_{2} \mathrm{COOC}\left(\mathrm{CH}_{3}\right)_{3}(\mathbf{l}), \mathrm{CH}_{2} \mathrm{CONHC}\left(\mathrm{C}_{6} \mathrm{H}_{5}\right)_{3}(\mathbf{m})$, $\mathrm{CH}_{2}$-5-imidazolyl-1-C $\left(\mathrm{C}_{6} \mathrm{H}_{5}\right)_{3}(\mathbf{n}), \mathrm{H}(\mathbf{o}),\left(\mathrm{CH}_{2}\right)_{2} \mathrm{CONHC}\left(\mathrm{C}_{6} \mathrm{H}_{5}\right)_{3}(\mathbf{p})$

Regents and conditions: (i) $(\mathrm{HCHO})_{n}, \mathrm{KOH}, \mathrm{H}_{2} \mathrm{O}$, ice bath $\sim 50{ }^{\circ} \mathrm{C}, 70 \mathrm{~h}$; (ii) L-cystein hydrochloride, $2 \mathrm{~mol} / \mathrm{L} \mathrm{HCl} ; 50{ }^{\circ} \mathrm{C}, 50 \mathrm{~h}$; (iii) Boc $2 \mathrm{O}$, $\mathrm{KOH}, \mathrm{H}_{2} \mathrm{O}$, ice bath $\sim$ r.t., $20 \mathrm{~h}$; (iv) $\mathrm{CH}_{3} \mathrm{OH}, \mathrm{SOCl}_{2}$, ice bath $\sim$ r.t., 6 h; (v) Fmoc-AA-OH, HOBt, DIC or DCC, DIPEA, THF, ice bath.

图式 1 目标化合物的合成

Scheme 1 Synthesis of target compounds

碱以促进偶联反应的进行，但氨基酸在强碱性环境中易 消旋，因此选用能接受游离质子(有碱性)但不夺取 $\alpha$-质 子(空间体积大)的有机碱 $N, N$-二异丙基乙胺(DIPEA), 可较好避免手性氨基酸消旋. 其次, Fmoc-AA-OH 长时 间处于强碱性环境中易脱除 Fmoc 基团, 因此要尽量弱 化反应条件. 表 1 数据显示, 某些分子, 如 TM-c, TM-g 和 TM-p, 反应时间都长但是收率却低于 $43 \%$, 很可能 和副反应的发生相关. 最后, 氨基酸的侧链取代基对反 应收率也有一定的影响. 如氨基酸侧链的体积较大或者 保护基(如 Trt, Boc)体积很大, 目标产物的收率低于 46\%(如 TM-c, TM-e, TM-g, TM-n 和 TM-p), 反之对应 目标化合物的收率较高, 其中结构最简单的化合物 TM-a 收率最高 $(86 \%)$.

\section{2 生物活性}

在成都地奥制药集团新药研究中心对本研究合成 的 16 个化合物进行生物活性测定，测试结果见表 2 , 其 中过氧化物酶体增殖物激活受体反应元件(PPRE)激动 活性、 $\alpha$-葡萄糖苷酶-rat 抑制活性、DPP-4 抑制活性测 试中采用的阳性参照物分别为上市药物 Pioglitazone, Voglibose 以及 KR-62436. 绝大多数目标分子的 PPRE 激动活性很弱，只有化合物 TM-e(含有 Val 结构单元的 分子)的 PPRE 相对激动活性达到 40.69\% (Pioglitazone 的 PPRE 激动活性为 $100 \%$ ). 构效分析发现，氨基酸侧 链取代基 $\mathrm{R}$ 中含有苯环结构的分子活性都很差，侧链取 代基中含有空间体积很大的保护基团(Trt)的化合物

表 1 目标分子 TM 的制备

Table 1 Synthesis of the target molecules TM

\begin{tabular}{clccccccc}
\hline Compd. & \multicolumn{1}{c}{ Fmoc-AA-OH } & Coupling reagent & Ratio $^{a}$ & Scale $/ \mathrm{mmol}$ & Time $/ \mathrm{h}$ & Temp. $/{ }^{\circ} \mathrm{C}$ & Weight $/ \mathrm{g}$ & Yield/\% \\
\hline TM-a & Fmoc-Ala-OH & DCC & $1: 1.1: 1.2$ & 2 & 7 & 26 & 0.952 \\
TM-b & Fmoc-Phe-OH & DIC & $1: 1: 1.2$ & 2 & 22 & 17 & 0.846 & 69 \\
TM-c & Fmoc-Val-OH & DIC & $1: 1: 1.2$ & 1 & 18 & 20 & 0.227 & 39 \\
TM-d & Fmoc-Leu-OH & DIC & $1: 1: 1.2$ & 1 & 17 & 24 & 0.351 & 59 \\
TM-e & Fmoc-Ile-OH & DIC & $1: 1: 1.2$ & 1 & 6 & 23 & 0.550 & 46 \\
TM-f & Fmoc-Thr(tBu)-OH & DIC & $1: 1: 1.2$ & 2 & 6 & 26 & 0.945 & 74 \\
TM-g & Fmoc-Asp(OtBu)-OH & DCC & $1: 1: 1.2$ & 2 & 4.5 & 25 & 0.582 & 43 \\
TM-h & Fmoc-Lys(Boc)-OH & DCC & $1.05: 1: 1.2$ & 2 & 6.5 & 27 & 0.871 & 61 \\
TM-i & Fmoc-Met-OH & DCC & $1: 1.1: 1.2$ & 2 & 6.5 & 27 & 0.626 & 51 \\
TM-j & Fmoc-Pro-OH & DCC & $1: 1.1: 1.2$ & 2 & 5.5 & 24 & 0.883 & 76 \\
TM-k & Fmoc-Tyr(tBu)-OH & DCC & $1: 1.1: 1.2$ & 2 & 7 & 21 & 0.880 & 64 \\
TM-I & Fmoc-Glu(OtBu)-OH & DIC & $1: 1: 1.2$ & 3 & 8.5 & 17 & 0.915 & 54 \\
TM-m & Fmoc-Asn(Trt)-OH & DCC & $1: 1.1: 1.2$ & 2 & 5 & 19 & 1.013 & 60 \\
TM-n & Fmoc-His(Trt)-OH & DCC & $1: 1.1: 1.2$ & 2 & 6 & 20 & 0.776 & 45 \\
TM-o & Fmoc-Gly-OH & DIC & $1: 1: 1.2$ & 1 & 17 & 24 & 0.429 & 79 \\
TM-p & Fmoc-Gln(Trt)-OH & DCC & $1: 1.1: 1.2$ & 2 & 6 & 18 & 0.696 & 41 \\
\hline
\end{tabular}

${ }^{a}$ Ratio 表示 IM-4/Fmoc-AA-OH/Coupling reagent 的物质的量之比. 
(TM-m, TM-n, TM-p), 其 PPRE 相对激动活性均为负 值. 因此, 减小氨基酸侧链保护基体积、脱出侧链保护 基, 或者改变 Fmoc 基团为其他结构单元, 都是在后续 研究中值得尝试的分子设计策略.

所合成的目标分子之间的活性差别，与取代基 $\mathrm{R}$ 有 关. 取代基 $\mathrm{R}$ 对分子活性的影响, 主要与 $\mathrm{R}$ 的电性和空 间体积、以及 $\mathrm{R}$ 对分子溶解性的影响有关. 从表 2 中 PPRE相对激动活性数据看, $R$ 为烷基侧链的分子的活性 较 $\mathrm{R}$ 含有活性基团侧链的分子活性高(如 TM-e $>$ TM-k), R 中含有苯环侧链的分子(如 TM-n, TM-m, TM-p, TM-k, TM-b)的活性都很弱. 令人难以理解的 是, 含有甘氨酸(TM-o) 和丙氨酸(TM-a) 结构单元的分 子, 其侧链结构很小, 也表现出低活性. 我们采用分子 对接技术对 TM-a, TM-e, TM-n 几个活性典型的分子与 PPAR $\gamma$ 配体结合结构域 2PRG 蛋白进行 3D 对接, 其对 接图见图 4. 对比阳性对照物 Pioglitazone 的对接图, TM-a 分子侧链结构太小, 不能与配体蛋白的受体腔进 行有效结合; TM-n 有庞大的侧链结构, 与配体蛋白结 合的氢键很多, 但在受体腔中结合很差, 依然导致低活 性(出现负值); 而 TM-e 侧链基团大小适中, 结合模式 与参照物 pioglitazone 相似, 表现出比其他分子相对较 好的活性, 但是相对于 Pioglitazone, 其与受体腔的匹配 程度稍差, 活性还不够理想, 因此该类分子的 PPRE 活 性结构还有待继续探索.

从表 2 数据可以看出, 目标分子的 $\alpha$-葡萄糖苷酶抑 制活性和 DPP-4 抑制活性都很弱. 然而将三种活性数据 作图(图 5), 从中可以看出, 目标化合物在 $\alpha$-葡萄糖苷酶
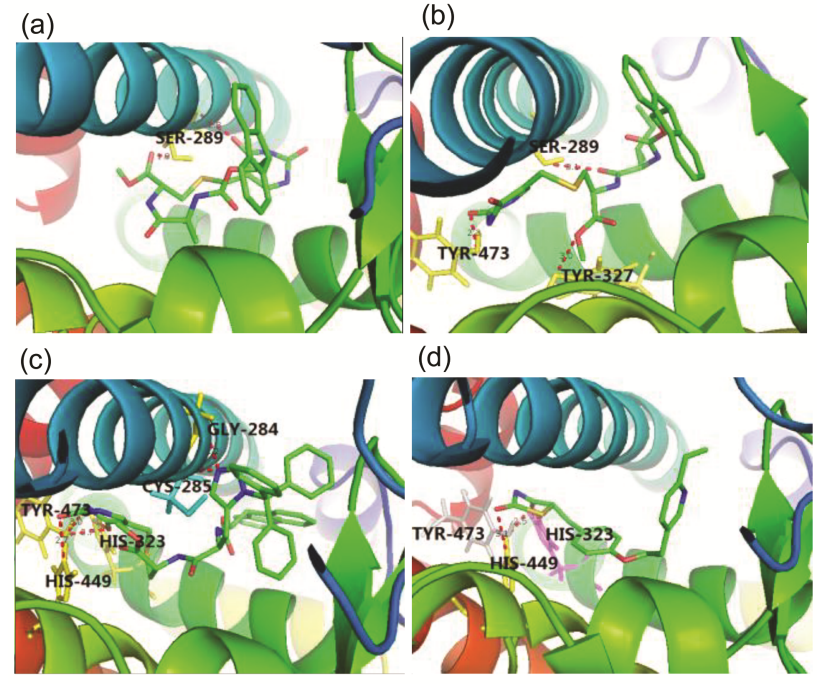

图 4 化合物 TM-a (a), TM-e (b), TM-n (c) 和 Pioglitazone (d) 与 PPAR $\gamma$ 配体结合结构域 $2 \mathrm{PRG}$ 的 3D 预测对接图

Figure 4 3D predicted binding modes of compounds TM-a (a), TM-e (b), TM-n (c) and pioglitazone (d) to 2PRG

抑制活性中的变化趋势刚好与 PPRE 激动活性、DPP-4 抑制活性的变化趋势相反, 这可能为多靶点药物的研究 提供了一种思路.

\section{2 结论}

以人体内天然碱基尿嘧啶和天然氨基酸半胱氨酸 为起始原料, 经过四步反应成功合成单体 $S$-胸腺嘧啶$L$-半胱氨酸甲酯盐酸盐; 以此单体作为氨基组分和不同 的 Fmoc 氨基酸进行偶联, 得到 16 个碱基修饰的二肽衍

表 2 目标化合物的 PPRE 相对激动活性、 $\alpha$-葡萄糖苷酶-rat 抑制活性及 DPP-4 抑制活性

Table 2 PPRE relative activation, $\alpha$-glucosidase inhibition and DPP-4 inhibition of target molecules

\begin{tabular}{|c|c|c|c|c|c|c|c|}
\hline Entry & AA & Conc. $/\left(\mu \mathrm{g} \cdot \mathrm{mL}^{-1}\right)$ & $\operatorname{cLog} P$ & tPSA & PPRE relative activation/\% & $\alpha$-Glucosidase inhibition/\% & DPP-4 inhibition/\% \\
\hline TM-a & Ala & 10.00 & 1.76 & 151.93 & -1.87 & 28.86 & -9.98 \\
\hline TM-b & Phe & 10.00 & 3.42 & 151.93 & 5.51 & 28.41 & -4.19 \\
\hline TM-c & Val & 10.00 & 2.65 & 151.93 & 27.08 & 17.91 & -7.39 \\
\hline TM-d & Leu & 10.00 & 3.02 & 151.93 & 13.72 & -15.66 & 0.00 \\
\hline TM-e & Ile & 10.00 & 3.10 & 151.93 & 40.69 & -14.19 & 14.42 \\
\hline TM-f & $\operatorname{Thr}(\mathrm{tBu})$ & 10.00 & 2.83 & 161.16 & 10.86 & 2.176 & -1.41 \\
\hline TM-g & $\mathrm{Asp}(\mathrm{OtBu})$ & 10.00 & 2.32 & 178.23 & 9.75 & 15.68 & -11.71 \\
\hline TM-h & Lys(Boc) & 10.00 & 1.45 & 181.03 & -3.72 & 20.20 & -16.50 \\
\hline TM-i & Met & 10.00 & 2.42 & 151.93 & 14.96 & -9.91 & 6.23 \\
\hline TM-j & Pro & 10.00 & 2.04 & 143.14 & -2.79 & -7.31 & 10.55 \\
\hline TM-k & $\operatorname{Tyr}(\mathrm{tBu})$ & 10.00 & 4.32 & 161.16 & 1.32 & -7.53 & 10.36 \\
\hline TM-I & Glu(OtBu) & 10.00 & 2.61 & 178.23 & 3.00 & -6.23 & 6.59 \\
\hline TM-m & Asn(Trt) & 10.00 & 5.70 & 181.03 & -9.62 & -2.78 & 4.69 \\
\hline TM-n & His(Trt) & 10.00 & 6.37 & 169.75 & -10.21 & -3.59 & 0.87 \\
\hline TM-о & Gly & 10.00 & 1.20 & 151.93 & 3.92 & -2.34 & -4.75 \\
\hline TM-p & $\mathrm{G} \ln (\mathrm{Trt})$ & 10.00 & 5.99 & 181.03 & -3.08 & -1.43 & 1.99 \\
\hline Pioglitazone & & 0.78 & 3.33 & 68.29 & 100.00 & - & - \\
\hline Voglibose & & 1.00 & -4.85 & 153.64 & - & 92.38 & - \\
\hline KR-62436 & & 0.30 & -1.03 & 117.2 & - & - & 73.11 \\
\hline
\end{tabular}




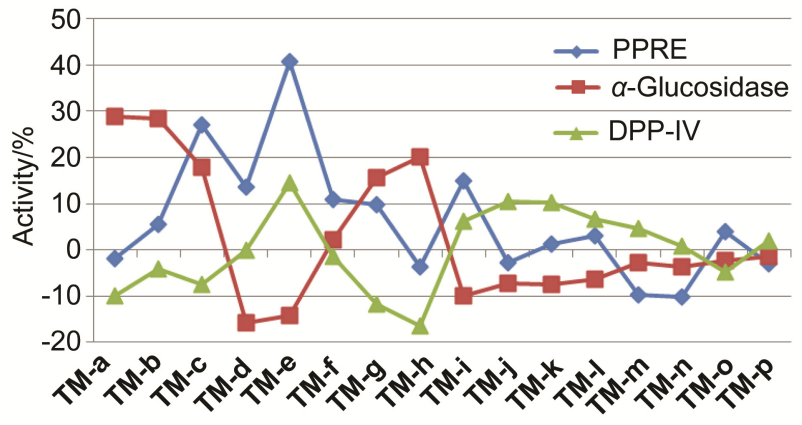

图 5 目标分子三种活性比较曲线图

Figure 5 Comparison graph of three kinds of target molecules activities

生物, 收率 $86 \%$ \% $39 \%$. 生物活性笁选结果显示, 二肽 衍生物的 PPRE 相对激动活性、 $\alpha$-葡萄糖苷酶的抑制活 性和二肽基肽酶抑制活性都很弱, 还需要进一步研究. 但作者发现该类分子表现出生物活性的选择性和多靶 向性, 为设计新型多肽多靶点药物的研究提供了新思 路.

\section{3 实验部分}

\section{1 仪器与试剂}

试剂规格及来源: 所用 Fmoc-AA-OH、半胱氨酸、 二环己基碳二亚胺(DCC)等(化学纯, 成都凯泰新技术有 限责任公司); HOBt (化学纯, 苏州天马医药有限公司), 二异丙基碳二亚胺(DIC)(分析纯, 山东淄博畅顺工贸有 限公司), $N, N$-二异丙基乙二胺(DIPEA)(化学纯, MerckSchuchardt, 浙江新德化工有限公司), 三乙胺(分析纯, 成都市科龙化工试剂厂), 其他常用溶剂规格及来源同 前.

\section{2 目标化合物 TM 的合成}

3.2.1 中间体 5-着弪甲基尿嘧啶(5-HmU, IM-1)的制备 $250 \mathrm{~mL}$ 圆底烧瓶加入 $\mathrm{KOH}(4.38 \mathrm{~g})$ 、水 $(150 \mathrm{~mL})$, 冰浴冷却, 分批加入尿嘧啶 $(11.23 \mathrm{~g}, 100 \mathrm{mmol})$, 搅拌 溶样; 几分钟后, 分批加入多聚甲醛 (3.602 g, 120 $\mathrm{mmol}$ ), 室温搅拌 $0.5 \mathrm{~h}$ 后, 置于 $50{ }^{\circ} \mathrm{C}$ 的水浴中, 继续摚 拌. 薄层色谱法(TLC)监测反应过程. 反应结束后, 取出 反应瓶, 冰浴下采用 $2 \mathrm{~mol} / \mathrm{L} \mathrm{HCl}$ 溶液调节 $\mathrm{pH}=7$, 搅 拌, 静置, 抽滤, 洗涤, 抽干. 合成结果及表征数据与参 考文献[24,25]一致.

3.2 .2 中间体 $S$-胸腺嘧啶 $-L$-半胱氨酸盐酸盐 $(L-\mathrm{HCl}$ ・ $\mathrm{H}-\mathrm{Cys}(\mathrm{T})-\mathrm{OH}, \mathbf{I M}-2)$ 的制备

在 $250 \mathrm{~mL}$ 圆底烧瓶中依次加入 $L$-半胱氨酸盐酸盐 (4.710 g, $30 \mathrm{mmol}$ )和 5-羊圣甲基尿嘧啶 (5-HmU) (4.260 g, $30 \mathrm{mmol}$ )后, 一次性加入浓度为 $2 \mathrm{~mol} / \mathrm{L}$ 的盐酸溶液 120 $\mathrm{mL}, 50{ }^{\circ} \mathrm{C}$ 搅拌反应, TLC 监测反应过程. 反应毕, 静置,
冷却, 析出白色固体, 抽滤, 干燥, 即得 $7.460 \mathrm{~g} \mathrm{~S}$-胸腺 嘧啶 $-L$ - 半胱氨酸盐酸盐 $[\mathrm{HCl} \cdot \mathrm{H}-\mathrm{Cys}(\mathrm{T})-\mathrm{OH}]^{[22]}$, 收率 $88 \%$. m.p. $239 \sim 241{ }^{\circ} \mathrm{C}$; IR (KBr) v: 3432, 3268, 3229, 3073, 2966, 2816, 1752, 1722, 1701, 1638, $1175 \mathrm{~cm}^{-1}$; API MS $m / z: 268.0[\mathrm{M}+\mathrm{Na}-\mathrm{HCl}]^{+}, 246.0[\mathrm{M}+\mathrm{H}-$ $\mathrm{HCl}]^{+}$.

3.2.3 中间体 $N$-叔丁氧羰基- $S$-胸腺嘧啶- $L$-半胱氨酸 (Boc-Cys(T)-OH, IM-3) 的制备

在 $250 \mathrm{~mL}$ 圆底瓶中加入 $\mathrm{HCl} \cdot \mathrm{H}-\mathrm{Cys}(\mathrm{T})-\mathrm{OH}(30$ $\mathrm{mmol}$ 和水 $50 \mathrm{~mL}$, 冰水浴冷却, 搅拌下缓慢加入溶有 氢氧化钾的水溶液 $100 \mathrm{~mL}$, 再分批加入 $\mathrm{Boc}_{2} \mathrm{O}(45$ $\mathrm{mmol})$, 加毕室温搅拌反应, TLC 监测反应过程, 反应过 程中适时用氢氧化钾水溶液调节反应液 $\mathrm{pH}$ 使其维持在 9 以上. 反应完毕后，乙醚萃取 $(30 \mathrm{~mL} \times 3)$, 水层冰水浴 冷却, 用浓度为 $2 \mathrm{~mol} / \mathrm{L}$ 的盐酸水溶液调节 $\mathrm{pH}$ 至 $3 \sim 4$, 有固体析出. 过滤, 滤饼用水洗涤 $(20 \mathrm{~mL} \times 2)$, 备用; 滤液用乙酸乙酯萃取 $(20 \mathrm{~mL} \times 4)$, 合并乙酸乙酯萃取液, 无水硫酸钠干燥，减压蒸馏回收溶剂，残余物加入 50 $\mathrm{mL}$ 石油醚(b.p. $60 \sim 90{ }^{\circ} \mathrm{C}$ ), 有固体析出. 冷藏过夜, 抽 滤, 所得滤饼与前述水洗后的滤饼合并, 干燥, 即得中 间体 Boc-Cys(T)-OH，白色固体 ${ }^{[22,23]}$.

3.2.4中间体 $S$-胸腺嘧啶- $L$ - 半胱氨酸甲酯盐酸盐 $\left(\mathrm{HCl} \cdot \mathrm{H}-\mathrm{Cys}(\mathrm{T})-\mathrm{OCH}_{3}, \mathbf{I M}-4\right)$ 的制备

在 $100 \mathrm{~mL}$ 圆底烧瓶中加入甲醇 $50 \mathrm{~mL}$, 冰盐浴冷 却, 搅拌下缓慢滴加二氯亚砜, 加毕搅拌 $30 \mathrm{~min}$, 再分 批加入 Boc-Cys(T)-OH, 加毕室温搅拌反应, TLC 监测 反应进程. 反应完毕后, 减压蒸馏回收溶剂, 残余物用 甲醇 $10 \mathrm{~mL}$ 分散后再减压旋蒸至干，重复 3 次以带出游 离 $\mathrm{HCl}$, 最后加入无水乙醚, 摚拌分散, 抽滤, 滤饼干 燥, 即得中间体 $\mathrm{HCl} \cdot \mathrm{H}-\mathrm{Cys}(\mathrm{T})-\mathrm{OCH}_{3}$, 白色粉末 ${ }^{[23]}$, m.p. 193.7 195.0 ${ }^{\circ} \mathrm{C}$; ESI MS m/z: $260.1\left([\mathrm{M}-\mathrm{Cl}]^{+}, 100\right)$.

\subsection{5 目标化合物 Fmoc-AA-Cys(T)- $\mathrm{OCH}_{3}$ (TM) 的制} 备

烧瓶中加入 $1 \mathrm{mmol} \mathrm{HCl} \cdot \mathrm{Cys}(\mathrm{T})-\mathrm{OCH}_{3} 、 1 \mathrm{~mL}$ 无水 四氢呋喃(THF)、1 mmol DIPEA, 冰浴冷却, 摚拌. 烧瓶 中加入 Fmoc-AA-OH (1 mmol)、HOBt $(0.162 \mathrm{~g}, 1.2$ $\mathrm{mmol}$ )、无水 THF $1.5 \mathrm{~mL}$ ，搅拌; $5 \mathrm{~min}$ 后，由移液枪注 射 DIC (0.2 mL, $1.2 \mathrm{mmol})$ 或加入 DCC (1.2 mmol), 冰浴 下搅拌. 活化 $0.5 \mathrm{~h}$ 后, 两液混合, TLC 监测反应进程. 反应完成后, 抽滤除去不溶物, 减压浓缩滤液去除 THF. 加入乙酸乙酯 $25 \mathrm{~mL}$, 搅拌尽量使其溶解, 再加入质量 分数为 $10 \%$ 柠檬酸水溶液 $20 \mathrm{~mL}$, 剧烈搅拌 $10 \mathrm{~min}$, 静 置, 分液. 乙酸乙酯层依次用 $0.5 \mathrm{~mol} / \mathrm{L} \mathrm{NaHCO} / \mathrm{H}_{2} \mathrm{O}$ 溶 液 $(15 \mathrm{~mL} \times 2)$ 、饱和 $\mathrm{NaCl}$ 溶液 $(15 \mathrm{~mL} \times 3)$ 洗涤; 所有水 层用乙酸乙酯 $(10 \mathrm{~mL} \times 2)$ 萃取, 合并有机层, 干燥后浓 
缩处理得到粗品, 再柱层析 (乙酸乙酯/石油醚或 $\left.\mathrm{CHCl}_{3} / \mathrm{CH}_{3} \mathrm{OH}\right)$ 得到目标分子 $\mathbf{T M}$.

芴甲氧羰基- $L$-丙氨酰- $S$ - (胸腺嘧啶) $-L$ - 半胱氨酸甲 酯(TM-a): 产率 86\%. m.p. $136 \sim 138{ }^{\circ} \mathrm{C} ;[\alpha]_{\mathrm{D}}^{17}-17.0(c$ $0.1, \mathrm{DMF}$ ); ${ }^{1} \mathrm{H}$ NMR (DMSO- $\left.d_{6}, 300 \mathrm{MHz}\right) \delta: 1.23$ (d, $J=$ $6.9 \mathrm{~Hz}, 3 \mathrm{H}, \mathrm{H}-25$ ), $2.73 \sim 2.85$ (m, 4H, H-15, H-16), 3.62 (s, 3H, H-24), $4.10 \sim 4.27$ (m, 4H, H-7, H-8, H-11), 4.47 4.49 (m, 1H, H-14), 7.32 7.41 (m, 4H, H-3, H-4), 7.53 (d, $J=7.59 \mathrm{~Hz}, 1 \mathrm{H}, \mathrm{H}-18), 7.72 \sim 7.83(\mathrm{~m}, 3 \mathrm{H}, \mathrm{H}-10$, H-5), 7.88 (d, $J=6.60 \mathrm{~Hz}, 2 \mathrm{H}, \mathrm{H}-2), 8.39$ (d, $J=7.50 \mathrm{~Hz}$, 1H, H-13), 10.84 (d, J=4.50 Hz, 1H, H-19), 11.16 (s, 1H, $\mathrm{H}-21) ;{ }^{13} \mathrm{C}$ NMR (DMSO- $\left.d_{6}, 75 \mathrm{MHz}\right) \delta: 172.9,171.1$, $168.2,165.9,163.7,151.3,139.4,128.9,127.7,127.3$, 127.1, 125.3, 121.4, 120.1 109.8, 65.7, 54.6, 52.1, 51.8, 46.7, 34.3, 32.2, 20.4; HRMS calcd for $\mathrm{C}_{27} \mathrm{H}_{28} \mathrm{~N}_{4} \mathrm{O}_{7} \mathrm{SNa}$ $[\mathrm{M}+\mathrm{Na}]^{+}$575.1571, found 575.1564.

芴甲氧羰基- $L$ - 苯丙氨酰- $S$-(胸腺嘧啶)- $L$ - 半胱氨酸 甲酯 (TM-b): 产率 $69 \%$. m.p. $203 \sim 204{ }^{\circ} \mathrm{C} ;[\alpha]_{D}^{17}$ -21.0 (c 0.1, DMF); ${ }^{1} \mathrm{H}$ NMR (DMSO- $d_{6}, 300 \mathrm{MHz}$ ) $\delta$ : $2.73 \sim 2.86(\mathrm{~m}, 4 \mathrm{H}, \mathrm{H}-15, \mathrm{H}-16), 2.88 \sim 3.00(\mathrm{~m}, 2 \mathrm{H}$, H-25), 3.64 (s, 3H, H-24), 4.11 4.27 (m, 4H, H-7, H-8, H-11), 4.54 (s, 1H, H-14), $7.18 \sim 7.43$ (m, 10H, H-3, H-4, H-18, H-27, H-28, H-29), 7.61 7.65 (m, 3H, H-10, H-5), $7.93(\mathrm{~d}, J=7.44 \mathrm{~Hz}, 2 \mathrm{H}, \mathrm{H}-2), 8.62(\mathrm{~d}, J=7.47 \mathrm{~Hz}, 1 \mathrm{H}$, H-13), 10.84 (d, J=4.53 Hz, 1H, H-19), 11.18 (s, 1H, $\mathrm{H}-21$ ); ${ }^{13} \mathrm{C}$ NMR (DMSO- $\left.d_{6}, 75 \mathrm{MHz}\right) \delta: 171.9,171.1$, 163.7, 155.8, 151.3, 143.8, 140.7, 139.4, 138.1, 129.3, 128.1, 127.7, 127.3, 126.3, 125.3, 120.1, 109.0, 65.7, 55.9, 52.2, 51.8, 46.6, 37.5, 32.2, 27.5; HRMS calcd for $\mathrm{C}_{33} \mathrm{H}_{32^{-}}$ $\mathrm{N}_{4} \mathrm{O}_{7} \mathrm{SNa}[\mathrm{M}+\mathrm{Na}]^{+}$651.1884, found 651.1880.

芴甲氧羰基- $L$ - 线氨酰- $S$ - (胸腺嘧啶) $L-$ - 半胱氨酸甲 酯(TM-c): 产率 39\%; m.p. 160 161 ${ }^{\circ} \mathrm{C} ;[\alpha]_{\mathrm{D}}^{17}-30.0$ (c 0.1, DMF); ${ }^{1} \mathrm{H}$ NMR (DMSO- $\left.d_{6}, 300 \mathrm{MHz}\right) \delta: 1.00$ (d, $J=$ $6.57 \mathrm{~Hz}, 6 \mathrm{H}, \mathrm{H}-26), 2.78 \sim 2.87$ (m, 5H, H-15, H-16, $\mathrm{H}-25$ ), 3.64 (s, 3H, H-24), 4.22 4.26 (m, 4H, H-7, H-8, $\mathrm{H}-11), 4.50$ (m, 1H, H-14), 7.30 7.44 (m, 5H, H-3, H-4, $\mathrm{H}-10), 7.73 \sim 7.77$ (m, 1H, H-18), 7.84 (d, $J=7.47 \mathrm{~Hz}, 2 \mathrm{H}$, $\mathrm{H}-2), 7.89$ (d, $J=7.41 \mathrm{~Hz}, 2 \mathrm{H}, \mathrm{H}-5), 8.46$ (d, $J=7.23 \mathrm{~Hz}$, $1 \mathrm{H}, \mathrm{H}-13) ;{ }^{13} \mathrm{C}$ NMR (DMSO- $\left.d_{6}, 75 \mathrm{MHz}\right) \delta: 166.8,166.5$, $163.6,156.8,151.2,139.4,139.0,137.4,129.5,128.9$, $127.3,121.4,120.0,109.7,109.2,59.4,54.1,48.6,34.3$, $31.2,27.5,23.3,18.5,17.2$; HRMS calcd for $\mathrm{C}_{29} \mathrm{H}_{32^{-}}$ $\mathrm{N}_{4} \mathrm{O}_{7} \mathrm{SNa}[\mathrm{M}+\mathrm{Na}]^{+}$603.1884, found 603.1890.

芴甲氧羰基- $L$ - 亮氨酰- $S$-(胸腺嘧啶)- $L$ - 半胱氨酸甲 酯(TM-d): 产率 59\%. m.p. $148 \sim 149{ }^{\circ} \mathrm{C} ;[\alpha]_{\mathrm{D}}^{17}-39.0(c$
0.1, DMF); ${ }^{1} \mathrm{H}$ NMR (DMSO- $\left.d_{6}, 300 \mathrm{MHz}\right) \delta: 1.00$ (d, $J=$ $6.51 \mathrm{~Hz}, 6 \mathrm{H}, \mathrm{H}-27), 1.41 \sim 1.47$ (m, 3H, H-26, H-25), $2.77 \sim 2.88$ (m, 4H, H-15, H-16), 3.63 (s, 3H, H-24), $4.22 \sim 4.26$ (m, 4H, H-7, H-8, H-11), 4.47 $4.54(\mathrm{~m}, 1 \mathrm{H}$, $\mathrm{H}-14), 7.34$ (t, $J=7.41 \mathrm{~Hz}, 4 \mathrm{H}, \mathrm{H}-3, \mathrm{H}-4), 7.43$ (d, $J=7.32$ $\mathrm{Hz}, 1 \mathrm{H}, \mathrm{H}-18), 7.71 \sim 7.74$ (m, 1H, H-10), 7.84 (d, $J=7.44$ Hz, 2H, H-5), 7.89 (d, $J=7.46 \mathrm{~Hz}, 2 \mathrm{H}, \mathrm{H}-2$ ), 8.42 (d, $J=$ $7.41 \mathrm{~Hz}, 1 \mathrm{H}, \mathrm{H}-13$ ); ${ }^{13} \mathrm{C}$ NMR (DMSO- $\left.d_{6}, 75 \mathrm{MHz}\right) \delta$ : $167.9,166.2,163.6,156.8,151.2,139.4,139.1,137.4$, $135.4,133.2,129.5,128.9,127.3,123.9,121.4,121.2$, 120.0, 109.7, 109.1, 54.5, 52.7, 48.6, 44.0, 34.5, 27.7, 23.4, 23.3, 23.1, 21.8; HRMS calcd for $\mathrm{C}_{30} \mathrm{H}_{34} \mathrm{~N}_{4} \mathrm{O}_{7} \mathrm{SNa}[\mathrm{M}+$ $\mathrm{Na}]^{+}$617.2040, found 617.2047.

芴甲氧羰基- $L$-异亮氨酰- $S$-(胸腺嘧啶)- $L$ - 半胱氨酸 甲酯(TM-e): 产率 46\%; m.p. 199 $201{ }^{\circ} \mathrm{C}$; $[\alpha]_{\mathrm{D}}^{17}-29.0$ (c 0.1, DMF); ${ }^{1} \mathrm{H}$ NMR (DMSO- $\left.d_{6}, 300 \mathrm{MHz}\right) \delta: 0.80 \sim$ 0.87 (m, 8H, H-28, H-26, H-27), 2.75 2.88 (m, 5H, H-15, H-16, H-25), 3.61 (s, 3H, H-24), 3.92 $3.99(\mathrm{~m}, 1 \mathrm{H}$, $\mathrm{H}-11), 4.21 \sim 4.26(\mathrm{~m}, 3 \mathrm{H}, \mathrm{H}-7, \mathrm{H}-8), 4.47 \sim 4.49(\mathrm{~m}, 1 \mathrm{H}$, $\mathrm{H}-14), 7.30 \sim 7.34$ (m, 1H, H-18), $7.39 \sim 7.44$ (m, 4H, H-3, $\mathrm{H}-4), 7.72 \sim 7.76(\mathrm{~m}, 1 \mathrm{H}, \mathrm{H}-10), 7.84(\mathrm{~d}, J=7.56 \mathrm{~Hz}, 2 \mathrm{H}$, H-2), 7.89 (d, $J=7.41 \mathrm{~Hz}, 2 \mathrm{H}, \mathrm{H}-5), 8.48$ (d, $J=7.29 \mathrm{~Hz}$, $1 \mathrm{H}, \mathrm{H}-13$ ); ${ }^{13} \mathrm{C}$ NMR (DMSO- $\left.d_{6}, 75 \mathrm{MHz}\right) \delta: 166.9,166.5$, $163.7,151.2,143.9,139.0,135.4,129.5,128.9,127.7$, $127.3,123.9,122.2,121.4,121.2,120.0,109.8,109.2$, 58.7, 54.1, 48.6, 38.0, 34.2, 27.6, 24.4, 15.0, 11.9; HRMS calcd for $\mathrm{C}_{30} \mathrm{H}_{34} \mathrm{~N}_{4} \mathrm{O}_{7} \mathrm{SNa}[\mathrm{M}+\mathrm{Na}]^{+}$617.2040, found 617.2040.

芴甲氧羰基- $L-(O$ - 叔丁基)-苏氨酰- $S$-(胸腺嘧啶)- $L$ 半胱氨酸甲酯(TM-f): 产率 $74 \%$, m.p. $157 \sim 159^{\circ} \mathrm{C}$; $[\alpha]_{\mathrm{D}}^{17}-12.0$ ( $c$ 0.1, DMF); ${ }^{1} \mathrm{H}$ NMR (DMSO- $d_{6}, 300 \mathrm{MHz}$ ) $\delta$ : 1.05 (d, $J=6.06 \mathrm{~Hz}, 3 \mathrm{H}, \mathrm{H}-26), 1.12$ (s, 9H, H-28), $2.81 \sim 2.88$ (m, 4H, H-15, H-16), 3.63 (s, 3H, H-24), $3.82 \sim 3.86(\mathrm{~m}, 1 \mathrm{H}, \mathrm{H}-25), 4.02 \sim 4.08(\mathrm{~m}, 1 \mathrm{H}, \mathrm{H}-11)$, $4.22 \sim 4.33(\mathrm{~m}, 3 \mathrm{H}, \mathrm{H}-7, \mathrm{H}-8), 4.52 \sim 4.54(\mathrm{~m}, 1 \mathrm{H}, \mathrm{H}-14)$, $7.29 \sim 7.44$ (m, 5H, H-3, H-4, H-18), $7.72 \sim 7.76(\mathrm{~m}, 3 \mathrm{H}$, H-10, H-5), 7.89 (d, $J=7.38 \mathrm{~Hz}, 2 \mathrm{H}, \mathrm{H}-2), 8.28$ (d, $J=$ $7.20 \mathrm{~Hz}, 1 \mathrm{H}, \mathrm{H}-13), 10.83$ (d, $J=4.59 \mathrm{~Hz}, 1 \mathrm{H}, \mathrm{H}-19$ ), 11.16 (s, $1 \mathrm{H}, \mathrm{H}-21) ;{ }^{13} \mathrm{C}$ NMR (DMSO- $\left.d_{6}, 75 \mathrm{MHz}\right) \delta$ : $170.9,169.8,163.7,155.9,151.2,143.9,143.7,140.7$, 139.4, 127.7, 127.1, 125.4, 120.1, 109.0, 73.9, 67.6, 65.8, 59.4, 52.1, 46.7, 32.5, 28.1, 27.4, 19.0; HRMS calcd for $\mathrm{C}_{32} \mathrm{H}_{38} \mathrm{~N}_{4} \mathrm{O}_{8} \mathrm{SNa}[\mathrm{M}+\mathrm{Na}]^{+}$661.2303, found 661.2306.

芴甲氧羰基 $-L-(O$ - 叔丁酯 $)$ - 天冬氨酰- $S$ - (胸腺嘧 啶)- $L$ - 半胱氨酸甲酯(TM-g): 产率 $43 \%$, m.p. 116 
$119{ }^{\circ} \mathrm{C} ;[\alpha]_{\mathrm{D}}^{17}-35.0$ (c 0.1, DMF); ${ }^{1} \mathrm{H}$ NMR (DMSO- $d_{6}$, $300 \mathrm{MHz}) \delta: 1.38$ (d, $J=7.16 \mathrm{~Hz}, 9 \mathrm{H}, \mathrm{H}-28), 2.64 \sim 2.84$ (m, 6H, H-25, H-15, H-16), 3.63 (s, 3H, H-24), 4.21 4.31 (m, 3H, H-7, H-8), 4.45 4.47 (m, 2H, H-14, H-11), 7.31 (t, $J=7.38 \mathrm{~Hz}, 2 \mathrm{H}, \mathrm{H}-3), 7.39 \sim 7.44$ (m, 3H, H-4, H-18), $7.65 \sim 7.71$ (m, 3H, H-10, H-5), 7.89 (d, $J=7.44 \mathrm{~Hz}, 2 \mathrm{H}$, H-2), 8.46 (d, $J=7.38 \mathrm{~Hz}, 1 \mathrm{H}, \mathrm{H}-13), 10.85$ (d, $J=4.83$ $\mathrm{Hz}, 1 \mathrm{H}, \mathrm{H}-19$ ), 11.18 (s, 1H, H-21); ${ }^{13} \mathrm{C}$ NMR (DMSO- $d_{6}$, $75 \mathrm{MHz}) \delta: 171.0,169.2,163.7,155.8,151.2,143.9$, $143.7,140.7,139.4,127.7,127.1,125.3,120.2,109.1$, 80.3, 65.8, 52.5, 52.2, 51.3, 46.7, 37.6, 32.1, 27.7, 27.4; HRMS calcd for $\mathrm{C}_{32} \mathrm{H}_{36} \mathrm{~N}_{4} \mathrm{O}_{9} \mathrm{SNa}[\mathrm{M}+\mathrm{Na}]^{+}$675.2095, found 675.2098 .

芴甲氧羰基-( $N$-叔丁氧羰基)- $L$-赖氨酰- $S$ - (胸腺嘧 啶)- $L$ - 半胱氨酸甲酯(TM-h): 产率 61\%. m.p. 131 $135{ }^{\circ} \mathrm{C} ;[\alpha]_{\mathrm{D}}^{17}-31.0$ (c 0.1, DMF); ${ }^{1} \mathrm{H}$ NMR (DMSO- $d_{6}$, $300 \mathrm{MHz}) \delta: 1.23 \sim 1.30(\mathrm{~m}, 2 \mathrm{H}, \mathrm{H}-26), 1.37(\mathrm{~s}, 9 \mathrm{H}$, $\mathrm{H}-31), 1.60 \sim 1.75(\mathrm{~m}, 4 \mathrm{H}, \mathrm{H}-27, \mathrm{H}-25), 2.77 \sim 2.89(\mathrm{~m}$, 6H, H-15, H-16, H-28), 3.63 (s, 3H, H-24), 4.10 4.12 (m, $1 \mathrm{H}, \mathrm{H}-7$ ), $4.22 \sim 4.28$ (m, 3H, H-8, H-11), 4.47 4.49 (m, 1H, H-14), 6.79 (m, 1H, H-29), 7.32 (t, $J=7.29 \mathrm{~Hz}, 2 \mathrm{H}$, $\mathrm{H}-3), 7.39 \sim 7.44$ (m, 3H, H-4, H-18), $7.48 \sim 7.50(\mathrm{~m}, 1 \mathrm{H}$, $\mathrm{H}-10), 7.72 \sim 7.74(\mathrm{~m}, 2 \mathrm{H}, \mathrm{H}-5), 7.89$ (d, $J=7.38 \mathrm{~Hz}, 2 \mathrm{H}$, H-2), 8.44 (d, $J=7.41 \mathrm{~Hz}, 1 \mathrm{H}, \mathrm{H}-13$ ), 10.86 (s, 1H, H-19), $11.18(\mathrm{~s}, 1 \mathrm{H}, \mathrm{H}-21) ;{ }^{13} \mathrm{C}$ NMR (DMSO- $\left.d_{6}, 75 \mathrm{MHz}\right) \delta$ : $172.3,171.1,163.7,155.6,151.2,143.9,142.6,140.7$, $139.4,137.4,128.9,127.6,127.3,127.0,125.3,121.4$, 120.0, 109.7, 109.0, 77.4, 67.0, 65.7, 54.4, 52.0, 51.8, 46.7, 32.2, 29.2, 28.3, 27.4, 25.1, 22.8; HRMS calcd for $\mathrm{C}_{35} \mathrm{H}_{43^{-}}$ $\mathrm{N}_{5} \mathrm{O}_{9} \mathrm{Na}[\mathrm{M}+\mathrm{Na}]^{+}$732.2674, found 732.2672.

芴甲氧羰基- $L$-蛋氨酰- $S$ - (胸腺嘧啶) $L-$ - 半胱氨酸甲 酯(TM-i): 产率 51\%. m.p. 195 198 ${ }^{\circ} \mathrm{C}$; $[\alpha]_{\mathrm{D}}^{17}-45.0(\mathrm{c}$ 0.1, DMF); ${ }^{1} \mathrm{H}$ NMR (DMSO- $\left.d_{6}, 300 \mathrm{MHz}\right) \delta: 1.85 \sim 1.90$ (m, 2H, H-25), 2.08 (s, 3H, H-27), 2.45 2.50 (m, $2 \mathrm{H}$, $\mathrm{H}-26), 2.78 \sim 2.89$ (m, 4H, H-15, H-16), 3.62 (s, 3H, $\mathrm{H}-24), 4.17 \sim 4.30$ (m, 4H, H-7, H-8, H-11), 4.47 4.49 (m, 1H, H-14), 7.32 (t, $J=7.26 \mathrm{~Hz}, 2 \mathrm{H}, \mathrm{H}-3$ ), $7.39 \sim 7.44$ (m, 3H, H-4, H-18), 7.60 (d, $J=8.13 \mathrm{~Hz}, 1 \mathrm{H}, \mathrm{H}-10), 7.73$ (d, $J=6.18 \mathrm{~Hz}, 2 \mathrm{H}, \mathrm{H}-5), 7.89$ (d, $J=7.38 \mathrm{~Hz}, 2 \mathrm{H}, \mathrm{H}-2)$, 8.47 (d, $J=7.32 \mathrm{~Hz}, 1 \mathrm{H}, \mathrm{H}-13), 10.84$ (d, $J=3.66 \mathrm{~Hz}, 1 \mathrm{H}$, $\mathrm{H}-19$ ), 11.17 (s, 1H, H-21); ${ }^{13} \mathrm{C}$ NMR (DMSO- $d_{6}, 75$ MHz) $\delta: 171.0,169.9,163.7,162.3,151.2,142.6,140.7$, $139.4,137.4,128.9,127.6,127.3,127.0,125.3,124.2$, 121.4, 120.0, 109.7, 109.0, 65.6, 55.9, 54.5, 52.2, 46.7, 35.7, 32.2, 30.8, 29.6, 14.6; HRMS calcd for $\mathrm{C}_{29} \mathrm{H}_{32-}$
$\mathrm{N}_{4} \mathrm{O}_{7} \mathrm{~S}_{2} \mathrm{Na}[\mathrm{M}+\mathrm{Na}]^{+}$635.1605, found 635.1611.

芴甲氧羰基 $-L$-脯氨酰- $S$-(胸腺嘧啶) $-L$ - 半胱氨酸甲 酯(TM-j): 产率 76\%. m.p. 106 107 ${ }^{\circ} \mathrm{C} ;[\alpha]_{\mathrm{D}}^{17}-56.0(c$ 0.1, DMF); ${ }^{1} \mathrm{H}$ NMR (DMSO- $\left.d_{6}, 300 \mathrm{MHz}\right) \delta: 1.77 \sim 1.92$ (m, 4H, H-25, H-26), 2.74 2.85 (m, 4H, H-15, H-16), $3.26 \sim 3.42$ (m, 2H, H-27), 3.63 (s, 3H, H-24), 4.18 4.45 (m, 5H, H-7, H-8, H-11, H-14), 7.35 7.41 (m, 5H, H-3, H-4, H-18), $7.59 \sim 7.69$ (m, 2H, H-5), 7.88 (d, $J=7.29 \mathrm{~Hz}$, 2H, H-2), 8.58 (d, J=7.77 Hz, 1H, H-13), 10.83 (s, 1H, $\mathrm{H}-19$ ), 11.17 (s, $1 \mathrm{H}, \mathrm{H}-21$ ); ${ }^{13} \mathrm{C}$ NMR (DMSO- $d_{6}, 75$ MHz) $\delta: 172.1,171.1,163.7,154.0,151.2,143.6,140.8$, 139.4, 127.7, 127.2, 125.5, 125.2, 120.1, 109.0, 79.2, 67.1, 59.6, 52.4, 52.0, 46.7, 32.2, 30.7, 30.0, 25.2; HRMS calcd for $\mathrm{C}_{29} \mathrm{H}_{30} \mathrm{~N}_{4} \mathrm{O}_{7} \mathrm{SNa}[\mathrm{M}+\mathrm{Na}]^{+}$601.1727, found 601.1734.

芴甲氧羰基-( $O$-叔丁基) $-L$ - 酪氨酰- $S$-(胸腺嘧啶)- $L$ 半胱氨酸甲酯(TM-k): 产率 $64 \%$. m.p. $133 \sim 135{ }^{\circ} \mathrm{C}$; $[\alpha]_{\mathrm{D}}^{17}-44.0$ (c 0.1, DMF); ${ }^{1} \mathrm{H}$ NMR (DMSO- $\left.d_{6}, 300 \mathrm{MHz}\right)$ $\delta: 1.17$ (s, 9H, H-31), 2.76 2.99 (m, 4H, H-15, H-16), $3.25 \sim 3.12$ (m, 2H, H-25), 3.64 (s, 3H, H-24), 4.10 4.15 (m, 3H, H-7, H-8), 4.28 4.32 (m, 1H, H-11), 4.52 4.53 (m, 1H, H-14), 6.80 (d, $J=7.05 \mathrm{~Hz}, 2 \mathrm{H}, \mathrm{H}-28), 7.22$ (d, $J=7.38 \mathrm{~Hz}, 2 \mathrm{H}, \mathrm{H}-27), 7.30$ (d, $J=6.89 \mathrm{~Hz}, 2 \mathrm{H}, \mathrm{H}-5)$, $7.39 \sim 7.42$ (m, 3H, H-3, H-18), 7.62 7.66 (m, 3H, H-4, $\mathrm{H}-10), 7.87$ (d, $J=6.54 \mathrm{~Hz}, 2 \mathrm{H}, \mathrm{H}-2), 8.61(\mathrm{~d}, J=6.03 \mathrm{~Hz}$, $1 \mathrm{H}, \mathrm{H}-13), 10.86$ (s, 1H, H-19), 11.19 (s, 1H, H-21); ${ }^{13} \mathrm{C}$ NMR (DMSO- $\left.d_{6}, 75 \mathrm{MHz}\right) \delta: 171.9,171.1,163.8,155.9$, $153.5,151.3,143.8,140.7,139.5,132.6,129.8,127.7$, 127.1, 125.4, 123.3, 120.1, 109.1, 79.2, 77.6, 65.8, 56.0, 52.5, 46.6, 36.9, 32.3, 28.5, 27.5; HRMS calcd for $\mathrm{C}_{37} \mathrm{H}_{40} \mathrm{~N}_{4} \mathrm{O}_{8} \mathrm{SNa}[\mathrm{M}+\mathrm{Na}]^{+} 723.2459$, found 723.2462 .

芴甲氧羰基 $-L-(O$ - 叔丁酯) - 谷氨酰- $S$-(胸腺嘧啶) $-L$ 半胱氨酸甲酯(TM-I): 产率 $54 \%$. m.p. $135 \sim 137{ }^{\circ} \mathrm{C}$; $[\alpha]_{\mathrm{D}}^{17}-43.0$ (c 0.1, DMF); ${ }^{1} \mathrm{H}$ NMR (DMSO- $\left.d_{6}, 300 \mathrm{MHz}\right)$ $\delta: 1.39$ (s, 9H, H-29), 2.08 2.12 (m, 2H, H-25), 2.23 2.28 (m, 2H, H-26), $2.74 \sim 2.88$ (m, 4H, H-15, H-16), 3.62 (s, 3H, H-24), 4.08 4.29 (m, 4H, H-7, H-8, H-11), 4.48 4.50 (m, 1H, H-14), 7.32 (t, $J=7.26 \mathrm{~Hz}, 2 \mathrm{H}, \mathrm{H}-4), 7.39 \sim$ 7.44 (m, 3H, H-5, H-18), 7.55 (d, $J=8.16 \mathrm{~Hz}, 1 \mathrm{H}, \mathrm{H}-10$ ), 7.73 (t, $J=5.97 \mathrm{~Hz}, 2 \mathrm{H}, \mathrm{H}-3), 7.89$ (d, $J=7.29 \mathrm{~Hz}, 2 \mathrm{H}$, H-2), 8.46 (d, $J=7.29 \mathrm{~Hz}, 1 \mathrm{H}, \mathrm{H}-13$ ), 10.84 (s, 1H, H-19), 11.17 (s, $1 \mathrm{H}, \mathrm{H}-21) ;{ }^{13} \mathrm{C}$ NMR (DMSO- $\left.d_{6}, 75 \mathrm{MHz}\right) \delta$ : $171.7,171.0,163.7,155.9,151.2,143.9,143.7,140.7$, 139.4, 127.6, 127.1, 125.3, 120.1, 109.0, 79.7, 67.0, 65.7, 53.6, 52.3, 52.1, 46.7, 31.2, 30.7, 27.5, 25.1; HRMS calcd for $\mathrm{C}_{33} \mathrm{H}_{38} \mathrm{~N}_{4} \mathrm{O}_{9} \mathrm{SNa}[\mathrm{M}+\mathrm{Na}]^{+}$689.2252, found 689.2253 . 
芴甲氧羰基-( $N$-三苯甲基)- $L$-天冬酰胺酰- $S$ - (胸腺嘧 啶)- $L$ - 半胱氨酸甲酯(TM-m): 产率 60\%. m.p. 155 $157{ }^{\circ} \mathrm{C} ;[\alpha]_{\mathrm{D}}^{17}-40.0$ (c 0.1, DMF); ${ }^{1} \mathrm{H}$ NMR (DMSO- $d_{6}$, $300 \mathrm{MHz}) \delta: 2.54 \sim 2.73(\mathrm{~m}, 2 \mathrm{H}, \mathrm{H}-25), 2.76 \sim 2.89(\mathrm{~m}$, 4H, H-15, H-16), 3.62 (s, 3H, H-24), 4.21 4.47 (m, 5H, H-7, H-8, H-11, H-14), 7.19 7.26 (m, 15H, H-30, H-31, $\mathrm{H}-32), 7.32$ (t, $J=7.44 \mathrm{~Hz}, 2 \mathrm{H}, \mathrm{H}-4), 7.41 \sim 7.43(\mathrm{~m}, 3 \mathrm{H}$, H-3, H-18), $7.68 \sim 7.76$ (m, 3H, H-10, H-5), 7.90 (d, $J=$ $7.41 \mathrm{~Hz}, 2 \mathrm{H}, \mathrm{H}-2), 8.46$ (d, J=7.20 Hz, 1H, H-13), 8.55 (s, 1H, H-27), 10.84 (s, 1H, H-19), 11.17 (s, 1H, H-21); ${ }^{13} \mathrm{C}$ NMR (DMSO- $\left.d_{6}, 75 \mathrm{MHz}\right) \delta: 171.1,163.7,154.0,151.3$, $144.8,143.8,140.8,139.4,128.6,127.7,127.5,127.1$, 126.4, 125.3, 120.2, 109.0, 69.4, 65.8, 52.1, 46.7, 30.7, 27.3; HRMS calcd for $\mathrm{C}_{47} \mathrm{H}_{43} \mathrm{~N}_{5} \mathrm{O}_{8} \mathrm{SNa}[\mathrm{M}+\mathrm{Na}]^{+}$ 860.2724, found 860.2733.

芴甲氧羰基-( $N^{\prime}$-三苯甲基)- $L$ - 组氨酰- $S$-(胸腺嘧 啶)- $L$-半胱氨酸甲酯(TM-n): 产率 $45 \%$. m.p. 163 $165{ }^{\circ} \mathrm{C} ;[\alpha]_{\mathrm{D}}^{17}-49.0$ (c 0.1, DMF); ${ }^{1} \mathrm{H}$ NMR (DMSO- $d_{6}$, $300 \mathrm{MHz}) \delta: 2.73 \sim 2.95$ (m, 6H, H-15, H-16, H-25), 3.60 (s, 3H, H-24), 4.14 4.50 (m, 5H, H-7, H-8, H-11, H-14), 6.90 (s, 1H, H-27), 7.06 7.42 (m, 21H, H-4, H-3, H-18, H-28, H-31, H-32, H-33), 7.57 (d, $J=8.07$ Hz, 1H, H-10), $7.67(\mathrm{~d}, J=7.26 \mathrm{~Hz}, 2 \mathrm{H}, \mathrm{H}-5), 7.90(\mathrm{~d}, J=7.41 \mathrm{~Hz}, 2 \mathrm{H}$, H-2), 8.54 (d, $J=6.87 \mathrm{~Hz}, 1 \mathrm{H}, \mathrm{H}-13$ ), 10.87 (s, 1H, H-19), $11.18(\mathrm{~s}, 1 \mathrm{H}, \mathrm{H}-21) ;{ }^{13} \mathrm{C}$ NMR (DMSO- $\left.d_{6}, 75 \mathrm{MHz}\right) \delta$ : $171.1,171.0,163.7,155.8,151.2,143.7,141.5,140.7$, $139.4,137.1,129.2,128.2,127.6,127.1,125.3,125.3$, 120.1, 119.7, 108.9, 75.5, 67.0, 52.4, 52.0, 46.6, 32.2, 30.7, 27.4, 25.1; HRMS calcd for $\mathrm{C}_{49} \mathrm{H}_{44} \mathrm{~N}_{6} \mathrm{O}_{7} \mathrm{SNa}[\mathrm{M}+\mathrm{Na}]^{+}$ 883.2884, found 883.2881.

芴甲氧羰基- $L$-甘氨酰- $S$ - (胸腺嘧啶) $-L$ - 半胱氨酸甲 酯(TM-o): 产率 79\%. m.p. 153 154 ${ }^{\circ} \mathrm{C} ;[\alpha]_{\mathrm{D}}^{17}-18.0(c$ 0.1, DMF); ${ }^{1} \mathrm{H}$ NMR (DMSO- $\left.d_{6}, 300 \mathrm{MHz}\right) \delta: 2.74 \sim 2.89$ (m, 4H, H-15, H-16), 3.64 (s, 3H, H-24), 3.68 (d, $J=5.58$ Hz, 2H, H-11), 4.20 4.30 (m, 3H, H-7, H-8), 4.51 4.55 (m, 1H, H-14), 7.33 (t, $J=7.32 \mathrm{~Hz}, 2 \mathrm{H}, \mathrm{H}-4), 7.40 \sim 7.45$ (m, 3H, H-3, H-18), 7.58 (t, $J=6.00 \mathrm{~Hz}, 1 \mathrm{H}, \mathrm{H}-10), 7.72$ (d, $J=7.29 \mathrm{~Hz}, 2 \mathrm{H}, \mathrm{H}-5), 7.90$ (d, $J=7.41 \mathrm{~Hz}, 2 \mathrm{H}, \mathrm{H}-2)$, 8.45 (d, $J=6.84 \mathrm{~Hz}, 1 \mathrm{H}, \mathrm{H}-13), 10.84$ (d, $J=5.01 \mathrm{~Hz}, 1 \mathrm{H}$, H-19), 11.19 (s, 1H, H-21); ${ }^{13} \mathrm{C}$ NMR (DMSO- $d_{6}, 100$ MHz) $\delta$ : 167.3, 166.4, 164.3, 151.9, 144.6, 143.3, 141.3, $141.1,140.0,138.1,130.1,129.6,128.0,124.6,122.0$, $121.8,120.6,110.4,109.7,55.2,54.5,49.3,45.1,35.4$, 31.3, 28.5; HRMS calcd for $\mathrm{C}_{26} \mathrm{H}_{26} \mathrm{~N}_{4} \mathrm{O}_{7} \mathrm{SNa}[\mathrm{M}+\mathrm{Na}]^{+}$ 561.1414, found 561.1411.
芴甲氧羰基-( $N$-三苯甲基)- $L$-谷氨酰胺酰- $S$ - (胸腺嘧 啶)- $L$ - 半胱氨酸甲酯(TM-p): 产率 $41 \%$. m.p. 143 $146{ }^{\circ} \mathrm{C} ;[\alpha]_{\mathrm{D}}^{17}-28.0$ (c 0.1, DMF); ${ }^{1} \mathrm{H}$ NMR (DMSO- $d_{6}$, $300 \mathrm{MHz}) \delta: 176 \sim 1.99(\mathrm{~m}, 2 \mathrm{H}, \mathrm{H}-25), 2.34 \sim 2.36(\mathrm{~m}$, $2 \mathrm{H}, \mathrm{H}-26$ ), $2.74 \sim 2.89$ (m, 4H, H-15, H-16), 3.61 (s, 3H, $\mathrm{H}-24), 4.04$ (t, $J=5.52 \mathrm{~Hz}, 1 \mathrm{H}, \mathrm{H}-7,), 4.23 \sim 4.30$ (m, $3 \mathrm{H}$, H-8, H-11), 4.49 (s, 1H, H-14), 7.17 7.35 (m, 17H, H-4, H-31, H-32, H-33), 7.40 7.51 (m, 4H, H-3, H-18, H-10), $7.74(\mathrm{~d}, J=7.17 \mathrm{~Hz}, 2 \mathrm{H}, \mathrm{H}-5), 7.90$ (d, $J=7.32 \mathrm{~Hz}, 2 \mathrm{H}$, H-2), 8.42 (d, $J=7.26 \mathrm{~Hz}, 1 \mathrm{H}, \mathrm{H}-13), 8.58$ (s, 1H, H-28), 10.85 (d, $J=4.86 \mathrm{~Hz}, 1 \mathrm{H}, \mathrm{H}-19), 11.19$ (s, $1 \mathrm{H}, \mathrm{H}-21) ;{ }^{13} \mathrm{C}$ NMR (DMSO- $\left.d_{6}, 100 \mathrm{MHz}\right) \delta$ : 171.9, 171.3, 170.9, 163.6, $155.9,151.1,144.8,143.7,140.6,139.3,128.4,127.6$, 127.4, 127.0, 126.2, 125.2, 120.0, 109.0, 69.1, 65.6, 59.7, $52.3,52.0,46.6,32.2,30.7,27.9,27.4$; HRMS calcd for $\mathrm{C}_{48} \mathrm{H}_{45} \mathrm{~N}_{5} \mathrm{O}_{8} \mathrm{SNa}[\mathrm{M}+\mathrm{Na}]^{+}$874.2881, found 874.2888.

\section{3 目标化合物的生物活性研究}

生物活性研究主要是抗糖尿病活性研究, 涉及 PPRE 激动活性、 $\alpha$-葡萄糖苷酶抑制活性及 DPP-4 抑制 活性的测定.

PPAR 反应元件(PPRE)激动活性笁选实验原理及实 验方法同参考文献[33]， $\alpha$-葡萄糖苷酶-rat 抑制剂篮选实 验原理及实验方法同参考文献 [33], 二肽基肽酶-IV (DPP-IV)抑制剂篮选实验原理及实验方法同参考文献 [34].

致谢 感谢成都地奥制药集团新药研究中心为本工作 中分子进行的生物活性测定.

辅助材料(Supporting Information) 中间体化合物 IM-2 及 IM-3 的合成结果和化合物 TM-a $~ T M-p ~$ 的 ${ }^{1} \mathrm{H}$ $\mathrm{NMR},{ }^{13} \mathrm{C}$ NMR 和 HRMS 图谱. 这些材料可以免费从 本刊网站(http://sioc-journal.cn/)上下载.

\section{References}

[1] Deshpande, A. D.; Harris-Hayes, M.; Schootman, M. Phys. Ther. 2008, 88, 1254.

[2] American Diabetes Association Diabetes Care, 2014, 37 (suppl. 1), S81.

[3] Karuranga, S.; Fernandes, J. R.; Huang, Y.; Malanda, B. International Diabetes Federation (IDF) Diabetes Atlas 8th, 2017, p. 10, www.diabetesatlas.org.

[4] Hemmingsen, B.; Sonne, D. P.; Metzendorf, M. I.; Richter, B Cochrane Database Syst. Rev. 2017, 5, CD012204.

[5] Nathan, K. T.; Ahmed-Sarwar, N.; Werner, P. Consult Pharm. 2016, $31,251$.

[6] Turner, N.; Zeng, X. Y.; Osborne, B.; Rogers, S.; Ye, J. M. Trends Pharmacol. Sci. 2016, 37, 379.

[7] Nissen, S. E.; Wolski, K. N. Engl. J. Med. 2007, 356, 2457.

[8] Faich, G. A.; Moseley, R. H. Drug Saf. 2001, $10,537$. 
[9] Riddle, M. C. Diabetes Care 2017, 40, 629.

[10] Leonard, C. E.; Hennessy, S.; Han, X.; Siscovick, D. S.; Flory, J. H.; Deo, $\quad$ R. Trends Endocrinol. Metab. 2017, 28, 561.

[11] Kajbaf, F.; Lalau, J. D. Pharmacoepidemiol. Drug Saf. 2014, 23, 1123.

[12] Richter, B.; Bandeira-Echtler, E.; Bergerhoff, K.; Lerch, C. L. Cochrane Database Syst. Rev. 2008, 16, CD006739.

[13] Nathan, D. M.; Buse, J. B.; Davidson, M. B.; Ferrannini, E.; Holman, R. R.; Sherwin, R.; Zinman, B. Diabetes Care 2009, 32, 193.

[14] Chen, Y. Technol. Econom. Guide 2016, 32, 100 (in Chinese). (陈俣，科技经济导刊, 2016, 32, 100.)

[15] Zhang, W.; Song, J. Q.; Zhang, B. Z.; Yang, W. L.; Wang, R. Sci. Sin.: Chim. 2013, 43, 941 (in Chinese) (张伟, 宋竟婧, 张邦治, 杨雯乐, 王锐, 中国科学: 化学, 2013, 43, 941.)

[16] Lee, K.; Boovanahalli, S. K.; Nam, K. Y.; Kang, S. U.; Lee, M.; Phan, J.; Wu, L.; Waugh, D. S.; Zhang, Z. Y.; No, K. T.; Lee, J. J.; Burke, T. R. Jr. Bioorg. Med. Chem. 2005, 15, 4037.

[17] Fu, H.; Park, J.; Pei, D. Biochemistry 2002, 41, 10700.

[18] Larsen, S. D.; Stevens, F. C.; Lindberg, T. J.; Bodnar, P. M.; O'Sullivan, T. J.; Schostarez, H. J.; Palazuk, B. J.; Bleasdale, J. E. Bioorg. Med. Chem. Lett. 2003, 13, 971.

[19] Zhang, D. Y.; Shao, W. F.; Liu, Z. H.; Liu, Y. L.; Huang, Y. W. J. Tea Sci. 2009, 29, 41 (in Chinese). (张冬英, 邵宛芳, 刘仲华, 刘亚林, 黄业伟, 茶叶科学, 2009, 29, 41.)

[20] Lee, B.; Shi, L.; Kassel, D. B.; Asakawa, T.; Takeuchi, K.; Christopher, R. J. Eur. J. Pharmacol. 2008, 589, 306

[21] Deng, X. Y.; Han, L.; Zhou, J. P.; Zhang, H. B.; Li, Q. Bioorg. Chem. 2017, 75, 357.

[22] Ma, L. J.; Zhang, G. L.; Chen, S. Y.; Wu, B.; You, J. S.; Xia, C. Q.; Yu, X. Q. J. Pept. Sci. 2005, 11, 812

[23] Tang, X. M.; Tang, G. X.; Wang, H.; Luo, L. F.; Yang, D. C. Bull.
Chem. Soc. Ethiop. 2012, 26, 415.

[24] Kong, L. Q.; Zhao, J.; Fan, L.; Yang, D. C. Chin. Chem. Lett. 2009, 20,314

[25] Zhao, J.; Kong, L. Q.; Fan, L.; Yang, D. C. J. Southwest Univ. (Nat. Sci.) 2008, 30, 54 (in Chinese) (赵晋, 孔令强, 范莉, 杨大成, 西南大学学报(自然科学版), 2008, 30, 54.)

[26] Yang, D. C.; Fan, L. Chem. Res. Appl. 2003, 15, 498 (in Chinese). (杨大成，范莉，化学研究与应用, 2003, 15, 498.)

[27] Yang, D. C.; Fan, L.; Zhong, Y. G. Chin. J. Org. Chem. 2003, 23 , 493 (in Chinese) (杨大成，范莉，钟裕国，有机化学, 2003, 23, 493.)

[28] Sun, X. L.; Fan, L.; Tang, X. M.; Yang, D. C. J. Org. Chem. Res. 2016, 4, 23 (in Chinese).

(孙晓丽, 范莉, 唐雪梅, 杨大成, 有机化学研究, 2016, 4, 23.)

[29] Wang, G. B.; Wang, L. F.; Li, C. Z.; Sun, J.; Zhou, G. M.; Yang, D. C. Res. Chem. Intermed. 2012, 38, 77.

[30] Yang, L.; Yan, J. F.; Fan, L.; Chen, X.; Shangguan, R. Y.; Wang, L. F.; Yang, D. C. Chin. J. Org. Chem. 2012, 32, 1908 (in Chinese) (杨龙, 晏菊芳, 范莉, 陈欣, 上官瑞燕, 汪林发, 杨大成, 有机 化学, 2012, 32, 1908.)

[31] Yang, Y.; Yan, J. F.; Fan, L.; Chen, X.; Jiang, L.; Yang, D. C. Acta Pharm. Sin. 2012, 47, 1630 (in Chinese). (杨艳, 晏菊芳, 范莉, 陈欣, 蒋理, 杨大成, 药学学报, 2012, 47, 1630.)

[32] Tang, X. M.; Fan, L.; Yu, H. X.; Liao, Y. H.; Yang, D. C. Chin. J. Org. Chem. 2009, 29, 595 (in Chinese). (唐雪梅，范莉，于红霞，廖玉华，杨大成，有机化学，2009，29， 595.)

[33] Tang, G. X.; Yan, J. F.; Fan, L.; Song, X. L.; Jiang, L.; Luo, L. F.; Yang, D. C. Sci. China, Chem. 2013, 56, 490.

[34] Zhang, X. H.; Yan, J. F.; Fan, L.; Wang, G. B.; Yang, D. C. Acta Pharm. Sin. B 2011, 1, 100 .

(Zhao, C.) 